chromosomes. Furthermore, among the candidate nuclear interaction partners we identified several mRNP members and proteins involved in mRNA processing and export, and these hits we could confirm by protein co-immunoprecipitation. Conclusions: Mass spectrometry analysis verified by protein co-immunoprecipitation suggests that Moesin's function is related to the NXF2-mediated mRNA export pathway as a possible new binding partner of the Mediator complex. In vitro assays will further confirm these protein interactions.

doi: http://dx.doi.org/10.7124/bc.0009EA

\section{L-1. Mutations in different domains of lamin A change the mechanical properties of the nucleus}

$\underline{\text { S. Lavrushkina }}^{1}$, N. Ovsiannikova ${ }^{1}$, A. Yudina $^{1}$, V. Kolmogorov ${ }^{1,3,4}$, P. Gorelkin ${ }^{3,5,8}$, O. Strelkova ${ }^{3}$, O. Zhironkina ${ }^{3}$, K. Perepelina ${ }^{6,7}$, A. Malashicheva ${ }^{6,7}$, I. Kireev ${ }^{1,2}$

${ }^{1}$ Lomonosov Moscow State University; ${ }^{2}$ National
Medical Research Center for Obstetrics, Gynecol-
ogy and Perinatology of Ministry of Healthcare of
Russian Federation, ${ }^{3}$ NanoProfiling LLC, Skolkovo
Innovation Centre; ${ }^{4}$ National University of Scence
and Technology «MISiS»; ${ }^{5}$ Medical Nanotechnol-
ogy LLC, Skolkovo Innovation Centre, Moscow,
Russia; ${ }^{6}$ St. Petersburg State Universityand; ${ }^{7}$ Labo-
ratory of Molecular Cardiology, Almazov National
Medical Research Center, St. Petersburg, Russia;
${ }^{8}$ ICAPPIC Limited, London, UK.
blodemwold@gmail.com

The rigid skeleton of cell nucleus, nuclear lamina (NL) consists mostly of A- and B-type lamins. Such structure maintains the nuclear shape and size and also provides mechanical link between nucleoplasm and cytoplasm. Mutations of structural components of NL are the cause of a wide group of diseases - laminopathies. According to "mechanical" hypothesis, this may be due to a disturbance of the mutant protein polymerization, as shown by the in vitro experiments. How NL assembly occurs in living cells is still unclear. Our aims were to visualize the structure of the nucleoskeleton in normal and pathological states and to estimate the mechanical properties of the nuclear envelope (NE) in living cells. In our work we used different types of cells: smooth muscle myocytes, cardiac progenitor cells, rat cardiomyocyte and human fibrosarcoma cells expressing wt lamin A or its mutant forms (G465D, R471C, R482L, R527C) fused to GFP. The resistance of NE of such cells to mechanical stress was studied by treating them with $15 / 30 \%$ Hanks' solution. The effect of a hypo-osmotic shock causes a mechanical stress inside the nucleus that leads to the formation of stable protrusions of NE - induced nuclear buds allowing us to indirectly estimate determined changes in the NE mechanical properties. Mechanical properties of NE were also measured with a scanning ion-conductance microscope (SICM), which allows to obtain stiffness index with high spatial resolution. In parallel, structural organization of NL was analyzed by structured illumination microscopy. We found the expansion of the distance between the NL microdomains are increased in nuclei containing mutant proteins. Furthermore, before treatment with a hypotonic solution, local disarrangement of the NL and nuclear asymmetry were observed in some 
cells. The use of SICM showed the dependence of the nucleus mechanical properties on the amount of lamin A and its isoforms. Therefore, the mechanical properties of the NE have tight relationship with molecular composition of NL. Thus, the cell nuclei expressing the additional lamin A gene were found to be 1.3 times more rigid than the wild-type cell nuclei, and changes in the structure of lamin A lead to improper organization of the nuclear skeleton. These may be due to the polymerization abnormality of dimers and decreased network stability and/or sequestration of normal protein.

The research was supported by Russian Science Fund № 17-15-01290.

doi: http://dx.doi.org/10.7124/bc.0009EB

\section{L-2. Irradiation by $\gamma$-rays reduces the level of $\mathrm{H3S10}$ phosphorylation and weakens the G2 phase-dependent interaction between $\mathrm{H3S10}$ phosphorylation and $\gamma \mathrm{H} 2 \mathrm{AX}$}

E. Bartova ${ }^{1}$, G. Lochmanova 2 , S. Legar$\underline{\text { tova }}^{1}$, J. Suchankova ${ }^{1}$, R. Fedr ${ }^{1}$, J. Krejci ${ }^{1}$, S. Kozubek ${ }^{1}$, I. Raska ${ }^{4}$, Z. Zdrahal ${ }^{2,3}$

${ }^{1}$ Institute of Biophysics of the Czech Academy of Sciences, Královopolská 135, 612 65, Brno, Czech Republic; ${ }^{2}$ Central European Institute of Technology (CEITEC), Kamenice 753/5, 625 00, Brno, Czech Republic; ${ }^{3}$ National Centre for Biomolecular Research, Kamenice 753/5, 625 00, Brno, Czech Republic; ${ }^{4}$ Institute of Biology and Medical Genetics, First Faculty of Medicine, Charles University and General University Hospital in Prague, Czech Republic legartova@ibp.cz
A mutual balance between specific histone markers is essential for optimal DNA repair. For example, the epigenetic factors HDAC1 and HDAC2 play a crucial regulating role in the DNA damage response [1]. To show how HDAC1 depletion in mouse embryonic stem cells (mESCs) can affect the histone signatures after being exposed to $\gamma$-rays, we used mass spectrometry, western blotting, immunohistochemistry, and advanced confocal microscopy. We mainly compared the epigenetic profiles of histones $\mathrm{H} 3$ and $\mathrm{H} 4$ in wild-type (wt) and HDAC1 double-knockout (dn) ES cells exposed to $\gamma$-rays. We observed that a decrease in H3S10 phosphorylation is a hallmark of $\gamma$-irradiated mESCs. However, radiation-induced changes in H3S10 phosphorylation (H3S10ph) were not affected by HDAC1 depletion. We found, as well a decrease in H3K9me3 induced by $\gamma$-irradiation but no difference at the level of HP1 $\beta$. These results fit well with the work published by [2] showing that all HP1 isoforms are recruited to UVinduced DNA lesions independently of H3K9me3. Changes in H3S10ph were likely caused by a $\gamma$-radiation-induced decrease in the number of cells in the G1 phase, characterized by no interaction between $\mathrm{H} 3 \mathrm{~S} 10 \mathrm{ph}$ and $\gamma \mathrm{H} 2 \mathrm{AX}$. However, an interaction of such modified histones we found in the G2 phase of the cell cycle. Together, our data show that even though H3S10ph is not directly involved in DNA repair, radiation-induced changes in the cell cycle can affect the function of H3S10ph.

This work was supported by the Czech Science Foundation (grant number: 18-07384S). The work was also supported by Strategie AV21, program Qualitas, from the Center of Epigenetics (ICO: 68081707). The CIISB research infrastructure project LM2015043 funded by MEYS CR is 